Borrelia miyamotoi sensu lato seroreactivity and seroprevalence in the northeastern United States. Emerg Infect Dis. 2014;20:1183-90. http://dx.doi.org/10.3201/eid2007.131587

4. Zhai B, Niu Q, Yang J, Liu Z, Liu J, Yin H, et al. Identification and molecular survey of Borrelia burgdorferi sensu lato in sika deer (Cervus nippon) from Jilin Province, north-eastern China. Acta Trop. 2017;166:54-7. http://dx.doi.org/10.1016/ j.actatropica.2016.11.002

5. Clark KL, Leydet B, Hartman S. Lyme borreliosis in human patients in Florida and Georgia, USA. Int J Med Sci. 2013; 10:915-31. http://dx.doi.org/10.7150/ijms.6273

6. Fomenko NV, Livanova NN, Borgoiakov VI, Kozlova IV, Shulaǐkina IV, Pukhovskaia NM, et al. Detection of Borrelia miyamotoi in ticks Ixodes persulcatus from Russia [in Russian]. Parazitologiia. 2010;44:201-11.

7. Liang FT, Aberer E, Cinco M, Gern L, Hu CM, Lobet YN, et al. Antigenic conservation of an immunodominant invariable region of the VlsE lipoprotein among European pathogenic genospecies of Borrelia burgdorferi SL. J Infect Dis. 2000;182:1455-62. http://dx.doi.org/10.1086/315862

8. Fonville M, Friesema IH, Hengeveld PD, Docters van Leeuwen A, Jahfari S, Harms MG, et al. Human exposure to tickborne relapsing fever spirochete Borrelia miyamotoi, the Netherlands. Emerg Infect Dis. 2014;20:1244-5. http://dx.doi.org/10.3201/eid2007.131525

9. Krause PJ, Fish D, Narasimhan S, Barbour AG. Borrelia miyamotoi infection in nature and in humans. Clin Microbiol Infect. 2015;21:631-9. http://dx.doi.org/10.1016/j.cmi.2015.02.006

10. Han S, Hickling GJ, Tsao JI. High prevalence of Borrelia miyamotoi among adult blacklegged ticks from white-tailed deer. Emerg Infect Dis. 2016;22:316-8. http://dx.doi.org/10.3201/ eid2202.151218

Address for correspondence: Zhangping Yang, Yangzhou University College of Animal Science and Technology, 12 East Wenhui Rd, Yangzhou, China, 225009; email: yzp@yzu.edu.cn; Chengming Wang, 1130 Wire Rd, College of Veterinary Medicine, Auburn University, Auburn, AL 36849, USA; email: wangche@auburn.edu

\section{Rickettsia asembonensis Characterization by Multilocus Sequence Typing of Complete Genes, Peru}

\author{
Steev Loyola, Carmen Flores-Mendoza, \\ Armando Torre, Claudine Kocher, \\ Melanie Melendrez, Alison Luce-Fedrow, \\ Alice N. Maina, Allen L. Richards, Mariana Leguia \\ Author affiliations: US Naval Medical Research Unit No. 6, Lima, \\ Peru (S. Loyola, C. Flores-Mendoza, A. Torre, C. Kocher, M. \\ Leguia); Pontificia Universidad Católica del Perú, Lima (A. Torre,
}

M. Leguia); Walter Reed Army Institute of Research, Silver Spring, Maryland, USA (M. Melendrez); Naval Medical Research Center, Silver Spring (A. Luce-Fedrow, A.N. Maina, A.L. Richards)

DOI: https://doi.org/10.3201/eid2405.170323

While studying rickettsial infections in Peru, we detected Rickettsia asembonensis in fleas from domestic animals. We characterized 5 complete genomic regions $(17 \mathrm{kDa}$, gltA, ompA, ompB, and sca4) and conducted multilocus sequence typing and phylogenetic analyses. The molecular isolate from Peru is distinct from the original $R$. asembonensis strain from Kenya.

$R$ ickettsia asembonensis belongs to a group of $R$. felislike organisms (RFLOs) that are similar, yet distinct, from their closest known relative, $R$. felis $(1,2)$. Although $R$. felis causes disease in humans (3), the pathogenicity of RFLOs remains unknown $(1,4,5)$. $R$. asembonensis was initially identified in domestic fleas from Kenya (1). Subsequently, reports from the Americas, Asia, and Africa established that $R$. asembonensis is ubiquitous and closely associated with human habitats because of its arthropod hosts (4-7). However, reports of $R$. asembonensis rarely include robust genomic information needed to establish degrees of genetic diversity. Consequently, many rickettsial infections remain underdiagnosed, even when prevalence is high (8). We recently described $R$. asembonensis in multiple ectoparasites (Ctenocephalidesfelis fleas and Rhipicephalussanguineus ticks) collected in the Peruvian Amazon (9). Here, we detail multilocus sequence typing of a single molecular isolate using next-generation sequencing data for 5 complete genomic regions, including conserved $(17 \mathrm{kDa}$ and $g l t A)$ and variable (ompA, ompB, and sca4) genes.

The internal review board of the US Naval Medical Research Unit No. 6 and the Institutional Animal Care and Use Committee approved the study protocol in compliance with all applicable regulations. Genomic DNA was mechanically extracted from half of a single C. felis flea as described (9) and fragmented by Bioruptor (Diagenode, Denville, NJ, USA). Fragmented DNA served as template to prepare IonPGM libraries using IonPlus Fragment Library Kits (ThermoFisher, Lima, Peru) according to the manufacturer's directions. We conducted quality control using Bioanalyzer High Sensitivity chips (Agilent, Lima, Peru). We prepared libraries for sequencing using IonPGM Template OT2 200 Kits (ThermoFisher, Lima, Peru) and conducted sequencing on 318 chips using IonPGM Sequencing 200 Kits v2 (ThermoFisher). We processed raw data by reference mapping against NMRCii from Kenya (10). Of the 20,575,878 shotgun sequencing reads generated, $\approx 12 \%$ matched Rickettsiaceae.

Comparison of the consensus sequences we generated (GenBank accession nos. KY650696-KY650700) with those of strain NMRCii (GenBank accession no. 
Table. Multilocus sequence typing analysis of complete genes from Peru Rickettsia asembonensis molecular isolate VGD7*

\begin{tabular}{|c|c|c|c|c|c|}
\hline \multirow[b]{2}{*}{ Changes } & \multicolumn{2}{|c|}{ Conserved genes (GenBank accession no.) } & \multicolumn{3}{|c|}{ Variable genes (GenBank accession no.) } \\
\hline & 17-kDa (KY650696) & gltA (KY650697) & ompA (KY650698) & ompB (KY650699) & sca4 (KY650700) \\
\hline \multicolumn{6}{|l|}{ Genome } \\
\hline Complete ORF, nt & 480 & 1,314 & 5,076 & 4,947 & 3,033 \\
\hline Identity, \% & 100 & 99.8 & 99.9 & 100 & 99.8 \\
\hline Mutations & None & $\begin{array}{l}\text { C } 138 \mathrm{~T} \\
\mathrm{~T} 537 \mathrm{C} \\
\mathrm{A} 868 \mathrm{G}\end{array}$ & $\begin{array}{l}\text { T } 484 \mathrm{G} \\
\text { C } 828 \mathrm{~A} \\
\text { A } 3838 \mathrm{G} \\
\text { C } 4360 \mathrm{G} \\
\text { T } 4880 \mathrm{C}\end{array}$ & None & $\begin{array}{l}\text { T } 383 \mathrm{C} \\
\text { T } 807 \mathrm{C} \\
\mathrm{G} 1824 \mathrm{~T} \\
\mathrm{~A} 2260 \mathrm{G} \\
\mathrm{T} 2492 \mathrm{C}\end{array}$ \\
\hline Total changes & 0 & 3 & 5 & 0 & 5 \\
\hline \multicolumn{6}{|l|}{ Protein } \\
\hline Complete protein, aa & 160 & 438 & 1,692 & 1,649 & 1,011 \\
\hline Identity, \% & 100 & 99.8 & 99.8 & 100 & 99.6 \\
\hline Mutations & None & K 290 E & $\begin{array}{l}Y 162 \mathrm{D} \\
\mathrm{R} 1280 \mathrm{G} \\
\mathrm{L} 1454 \mathrm{~V} \\
\mathrm{~V} 1627 \mathrm{~A}\end{array}$ & None & $\begin{array}{c}\mathrm{L} 128 \mathrm{P} \\
\mathrm{Q} 608 \mathrm{H} \\
\mathrm{R} 754 \mathrm{G} \\
\text { I } 831 \mathrm{~T}\end{array}$ \\
\hline Total changes & 0 & 1 & 4 & 0 & 4 \\
\hline
\end{tabular}

JWSW01000078.1) (10) indicates high identity at the nucleotide $(99.8 \%-100.0 \%)$ and amino acid $(99.6-100.0 \%)$ levels (Table). As expected, conserved genes (17kDa and gltA) showed fewer substitutions than variable genes ( ompA, omp $B$, and $s c a 4)$. The 17-kDa gene exhibited no mutations along its 480-nt open reading frame (ORF), whereas the gltA gene exhibited 3 mutations along its 1,314-nt ORF. Two mutations in gltA encoded silent changes, whereas the third encoded a conservative lysine-to-glutamic acid change at position 290. In the variable group, ompB exhibited no mutations along its 4,947-nt ORF; ompA and sca 4 exhibited 5 each. ompA had 2 conservative changes (leucine-to-valine at position 1454 and valine-to-alanine at position 1627) and 2 nonconservative changes (tyrosine-toaspartic acid at position 162 and arginine-to-glycine at position 1280). sca 4 had 1 conservative change (glutamineto-histidine at position 608) and 3 nonconservative changes (leucine-to-proline at position 128, arginine-to-glycine at position 754, and isoleucine-to-threonine at position 831 ). On the basis of these data, we conclude that the Peru molecular isolate is distinct from the original Kenya strain.

To further characterize the Peru isolate, we conducted phylogenetic analysis using the conserved gltA gene. Although reference sequences are available for multiple Rickettsiaceae, $R$. asembonensis sequences are limited in number and length (online Technical Appendix Table, https:// wwwnc.cdc.gov/EID/article/24/5/17-0323-Techapp1.pdf). Nevertheless, we constructed a phylogenetic tree using almost the complete gltA gene (1,068 [81\%] nt of the ORF). As expected, the Peru isolate groups with RFLOs, including other $R$. asembonensis isolates and $R$. senegalensis (online Technical Appendix Figure, panel A). Construction of an additional tree using only $348 \mathrm{nt}$ of $g l t A$ sequence available for an increased number of isolates (online Technical Appendix Table) enabled us to confirm placement and relationship with other strains from the Americas (online Technical Appendix Figure, panel B). This tree focuses exclusively on the transitional group and includes partial $R$. asembonensis references from Brazil, Colombia, and Costa Rica that were not available for inclusion in the 1,068-nt gltA tree. The Peru isolate clearly groups with other American isolates, and this subgroup is distinct from the original Kenya strain.

$R$. asembonensis is a new species (2) with potential as a ubiquitous human pathogen. Despite worldwide distribution, whether $R$. asembonensis and other RFLOs are pathogenic to humans, as is their closest relative $R$. $f e$ lis, remains unknown. Complete genomic data, which are largely lacking from public repositories, are required to assess genetic diversity. Using next-generation sequencing, we generated complete sequences for 2 conserved (17 $\mathrm{kDa}$ and $g l t A)$ and 3 variable $(\operatorname{omp} A, \operatorname{omp} B$, and $s c a 4)$ genes of an $R$. asembonensis molecular isolate from Peru. Although characterization of 1 isolate is not sufficient to evaluate strain diversity within Peru, much less among American strains, these sequences represent a major contribution toward the expansion of availability of much needed genomic information. Our multilocus sequence typing and phylogenetic analyses indicate that the Peru isolate is closer to American strains than to the original strain from Kenya. Characterization of additional isolates, derived from a variety of ectoparasites in which $R$. asembonensis has been detected, is needed to further validate our findings and to conduct in-depth diversity studies. In turn, these results should help decrease the chronic underdiagnosis of rickettsial diseases throughout the Americas.

This work was supported by work unit no. $800000.82000 .25 \mathrm{~GB}$. B0016, Promis ID\#MLeguia-PO166-14, for FY2014, from the Global Emerging Infections Surveillance and Response System of the Armed Forces Health Surveillance Center. 


\section{About the Author}

Mr. Loyola is a researcher in the Genomics \& Pathogen

Discovery Unit at the US Naval Medical Research Unit No. 6, in Lima, Peru. His primary research interest is the characterization of new and emerging pathogens.

\section{References}

1. Jiang J, Maina AN, Knobel DL, Cleaveland S, Laudisoit A, Wamburu K, et al. Molecular detection of Rickettsia felis and Candidatus Rickettsia asemboensis in fleas from human habitats, Asembo, Kenya. Vector Borne Zoonotic Dis. 2013;13:550-8. http://dx.doi.org/10.1089/vbz.2012.1123

2. Maina AN, Luce-Fedrow A, Omulo S, Hang J, Chan TC, Ade F, et al. Isolation and characterization of a novel Rickettsia species (Rickettsia asembonensis sp. nov.) obtained from cat fleas (Ctenocephalides felis). Int J Syst Evol Microbiol. 2016;66:4512-7. http://dx.doi.org/10.1099/ijsem.0.001382

3. Pérez-Osorio CE, Zavala-Velázquez JE, Arias-León JJ, Zavala-Castro JE. Rickettsia felis as emergent global threat for humans. Emerg Infect Dis. 2008;14:1019-23. http://dx.doi.org/ 10.3201/eid1407.071656

4. Maina AN, Fogarty C, Krueger L, Macaluso KR, Odhiambo A, Nguyen K, et al. Rickettsial infections among Ctenocephalides felis and host animals during a flea-borne rickettsioses outbreak in Orange County, California. PLoS One. 2016;11:e0160604. http://dx.doi.org/10.1371/journal.pone.0160604

5. Troyo A, Moreira-Soto RD, Calderon-Arguedas Ó, Mata-Somarribas C, Ortiz-Tello J, Barbieri AR, et al. Detection of rickettsiae in fleas and ticks from areas of Costa Rica with history of spotted fever group rickettsioses. Ticks Tick Borne Dis. 2016;7:1128-34. http:/dx.doi.org/10.1016/ j.ttbdis.2016.08.009

6. Kolo AO, Sibeko-Matjila KP, Maina AN, Richards AL, Knobel DL, Matjila PT. Molecular detection of zoonotic Rickettsiae and Anaplasma spp. in domestic dogs and their ectoparasites in Bushbuckridge, South Africa. Vector Borne Zoonotic Dis. 2016;16:245-52. http://dx.doi.org/10.1089/vbz.2015.1849

7. Oteo JA, Portillo A, Portero F, Zavala-Castro J, Venzal JM, Labruna MB. 'Candidatus Rickettsia asemboensis' and Wolbachia spp. in Ctenocephalides felis and Pulex irritans fleas removed from dogs in Ecuador. Parasit Vectors. 2014;7:455.

8. Forshey BM, Stewart A, Morrison AC, Gálvez H, Rocha C, Astete $\mathrm{H}$, et al. Epidemiology of spotted fever group and typhus group rickettsial infection in the Amazon basin of Peru. Am J Trop Med Hyg. 2010;82:683-90. http://dx.doi.org/10.4269/ ajtmh.2010.09-0355

9. Kocher C, Morrison AC, Leguia M, Loyola S, Castillo RM, Galvez HA, et al. Rickettsial disease in the Peruvian Amazon basin. PLoS Negl Trop Dis. 2016;10:e0004843. http://dx.doi.org/10.1371/ journal.pntd.0004843

10. Jima DD, Luce-Fedrow A, Yang Y, Maina AN, Snesrud EC, Otiang E, et al. Whole-genome sequence of "Candidatus Rickettsia asemboensis" strain NMRCii, isolated from fleas of western Kenya. Genome Announc. 2015;3:e0018-15. http://dx.doi.org/10.1128/ genomeA.00018-15

Address for correspondence: Mariana Leguia, Pontificia Universidad Católica del Perú, Av. Universitaria No. 1801, San Miguel, Lima, Perú; email: mariana.leguia@pucp.edu.pe

\section{Spontaneous Abortion Associated with Zika Virus Infection and Persistent Viremia}

\author{
Anna Goncé, Miguel J. Martínez, \\ Elena Marbán-Castro, Adela Saco, Anna Soler, \\ Maria Isabel Alvarez-Mora, Aida Peiro, \\ Verónica Gonzalo, Gillian Hale, Julu Bhatnagar, \\ Marta López, Sherif Zaki, Jaume Ordi, \\ Azucena Bardaji
}

Author affiliations: BCNatal-Barcelona Center of Maternal-Fetal and Neonatal Medicine, Barcelona, Spain (A. Goncé, M. López); ISGlobal, Hospital Clínic-Universitat de Barcelona, Barcelona (M.J. Martínez, E. Marbán-Castro, J. Ordi, A. Bardají); Hospital Clínic, Barcelona (M.J. Martínez, A. Saco, A. Peiro, V. Gonzalo, J. Ordi); Hospital Clínic, IDIBAPS and CIBERER, Barcelona (A. Soler, M.I. Alvarez-Mora); Centers for Disease Control and Prevention, Atlanta, Georgia, USA (G. Hale,

J. Bhatnagar, S. Zaki)

DOI: https://doi.org/10.3201/eid2405.171479

We report a case of spontaneous abortion associated with Zika virus infection in a pregnant woman who traveled from Spain to the Dominican Republic and developed a rash. Maternal Zika viremia persisted at least 31 days after onset of symptoms and 21 days after uterine evacuation.

$\mathrm{E}$ vidence regarding the association of Zika virus infection and pregnancy loss (spontaneous abortions and stillbirths) has been reported recently (1). Zika virus has been detected by reverse transcription PCR (RT-PCR) in brain tissue samples from stillborn infants and from placental tissue obtained from pregnancy losses $(2,3)$. We report a case of early pregnancy loss associated with Zika virus with evidence of persistent maternal viremia after uterine evacuation.

In mid-June 2016, a 22-year-old woman, who was in the seventh week of gestation, traveled from Spain to the Dominican Republic. Fifteen days after her arrival, she developed a mild macular rash and malaise that resolved after 3 days (Figure). One day after her return to Spain (at 10.5 weeks of pregnancy and 9 days after the onset of symptoms), a routine first-trimester prenatal scan showed an embryo without cardiac activity and a crown-rump length of $19 \mathrm{~mm}$, compatible with a pregnancy loss at an estimated gestational age of 8 weeks and 4 days (Figure). On July 5, 2016, a maternal serum sample tested positive for Zika virus by a commercial real-time RT-PCR with a cycle threshold $\left(\mathrm{C}_{\mathrm{t}}\right)$ value of 33 , and a urine sample was 